\title{
SUMMARY OF STUDIES ON THE BLUE-GREEN Autofluorescence AND Light Transmission OF THE OCULAR LENS
}

\author{
Jaap A. van Best and Esmeralda V. M. J. Kuppens \\ Leiden University Hospital, Department of Ophthalmology, Leiden, The Netherlands \\ (Paper JBO/LSF-04 received Mar. 6, 1996; revised manuscript received May 15, 1996; accepted for publication May 15, \\ 1996)
}

\begin{abstract}
This paper reviews previous work done to demonstrate the clinical relevance of the measurement of bluegreen autofluorescence and light transmission of the ocular lens $(\lambda=450$ to $550 \mathrm{~nm})$. These can be determined quantitatively with fluorophotometry in a few seconds. Autofluorescence and transmission values are determined in healthy volunteers, in patients with insulin-dependent diabetes mellitus, and in patients with untreated glaucoma or untreated ocular hypertension. The lens autofluorescence of healthy volunteers increased linearly and transmission decreased exponentially with age. Each year of diabetes induced an increase of autofluorescence equal to one extra year of age. Untreated glaucoma or ocular hypertension had no significant effect on lens autofluorescence and transmission. Increased autofluorescence and decreased transmission values in comparison with values of a healthy population are proved to be indicative for an increased risk of developing cataract and the clinical usefulness of these measures is demonstrated. Diabetes is a risk factor for developing cataract while untreated glaucoma or ocular hypertension is not. ( 1996 Society of Photo-Optical Instrumentation Engineers.
\end{abstract}

Keywords light scattering and fluorescence, ocular lens; light transmission; diabetes; glaucoma; UV-A.

\section{INTRODUCTION}

The healthy human lens is a coherent structure containing about $60 \%$ water and $38 \%$ proteins, mainly crystallins. The essential refractive and accommodative properties of the lens are derived from the variable curvature of this structure. ${ }^{1}$

Photo-oxidation of lens proteins by chronic UV, UV-A, or visible light results in oxidized forms of these proteins, which cross link to other proteins, causing opacities or pigment formation. These proteins remain in the eye for the entire human life span with little or no turnover., ${ }^{2,3}$ Consequently the resultant changes are cumulative, leading to agerelated coloration of the lens ${ }^{4}$ and presumably formation of the most common types of age-related cataract (cortical, nuclear, or posterior subcapsular cataract) ${ }^{5-7}$ Cataract is a partial or total opacification of the normally clear ocular lens, leading to reduced visual clarity and ultimately to blindness.

Concomitantly, fluorescent pigments accumulate in the human lens partly as by-products of the photo-oxidation of tryptophan and other substances. $^{8-10}$ As a result of this accumulation, aging of the lens is associated with an increase in fluorescence emission at $\lambda \approx 420 \mathrm{~nm}$, originating from

E-mail: vanbest@rullf2.leidenuniv.nl crystallins, and at $\lambda \approx 530 \mathrm{~nm}$, originating from yetunknown proteins. ${ }^{3}$

An increased lenticular autofluorescence at $\lambda \approx 530 \mathrm{~nm}$ and a decreased lens transmission at $\lambda=450$ to $550 \mathrm{~nm}$ have been proved to be indicative for an increased risk of cataract formation. ${ }^{11-13}$ Cataract is supposed to start gradually in a healthy lens, most likely resulting in a simultaneous increase in the autofluorescence. On the other hand, an increased lenticular autofluorescence at $\lambda \approx 420$ $\mathrm{nm}$ was found not to be an indicator of cataractogenesis. ${ }^{13}$

Few quantitative in vivo methods have been described that are based on a comparison of lens fluorescence with a fluorescence standard, ${ }^{14}$ on microdensitometric analysis of slit lamp photographs, ${ }^{15,16}$ or on scanning of the lens with a fluorophotometer. ${ }^{17}$ The studies described in this paper are a summary of earlier papers on the subject and concern measurements of lens autofluorescence and lens transmission $(\lambda=450$ to $550 \mathrm{~nm})$ performed with a scanning fluorophotometer. The measurements were done in patients with diabetes mellitus, primary open-angle glaucoma, and ocular hypertension since these patients are known to develop cataract prematurely. The results were compared with those in healthy volunteers. 


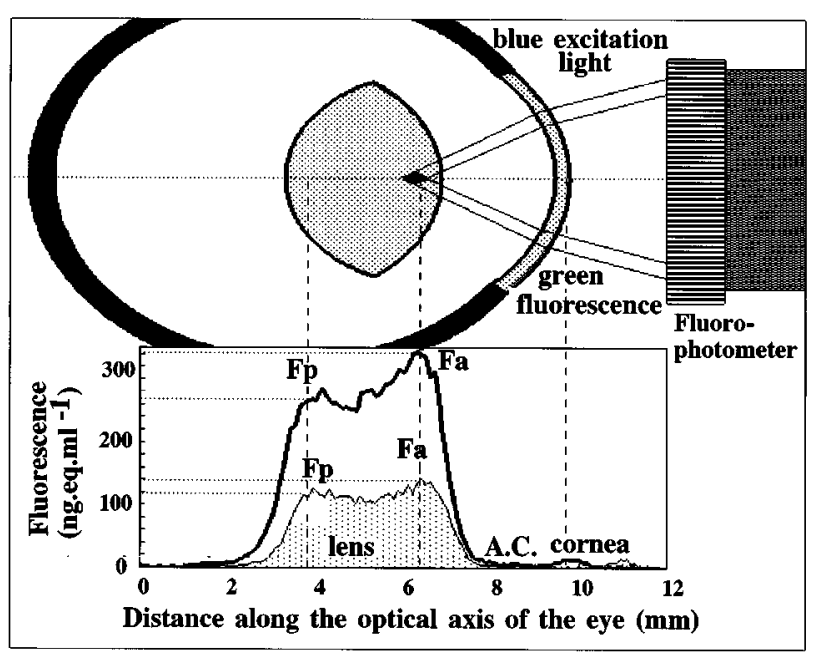

Fig. 1 Top: Principle of fluorophotometry. A beam of blue excitation light is projected in the eye and the green fluorescence is viewed along a "looking beam." In this way only fluorescence at the intercept of both "beams" (black diamond) is measured. This intercept is moved in steps from the posterior side of the lens up to the cornea. Bottom: The fluorescence profile obtained by stepwise fluorescence measurements along the optical axis of the eye. The upper thick and lower thin curves represent the fluorescence profile of healthy volunteers aged 59 and 27 years, respectively. $F_{a}$ and $F_{p}$ are the anterior and posterior lens fluorescence peaks, respectively. The transmission values calculated were 0.88 and 0.93 , respectively. A.C.: anterior chamber of the eye.

\section{METHODS}

\subsection{LENS AUTOFLUORESCENCE}

The lens autofluorescence measurements were performed with a commercially available scanning fluorophotometer (Fluorotron Master, Ocumetrix, Mountain View, California) fitted with a $\sim 2.5 \times$ magnifying lens ("anterior segment adapter") for detailed scanning of the lens, anterior chamber, and cornea. Measurements were done within $1 \mathrm{~min}$ without use of mydriatics, contact lens, or fluorescein. Autofluorescence excited by a beam of continuous light ( $\lambda=450$ to $500 \mathrm{~nm}$ ) was scanned along the optical axis of the eye in steps of $0.1 \mathrm{~mm}$ by moving the internal lens system of the fluorophotometer by a computer-controlled motor (Figure 1, top). The wavelengths of excitation and detected fluorescence light were set by color filters with peak transmissions at 490 and $530 \mathrm{~nm}$, respectively. The fluorescence intensity measured, expressed in equivalents of fluorescein concentration (ng. eq. $\mathrm{ml}^{-1}$ ), was recorded as a function of the distance in the eye (Figure 1, bottom). To avoid loss of excitation and fluorescent light by scatter and absorption within the lens, ${ }^{17-19}$ the peak value of axial autofluorescence in the anterior part of the lens was used as the parameter for lens fluorescence. The values measured were independent of the observer and the intraindividual variability was within $3 \%{ }^{3}$

\subsection{LENS TRANSMISSION}

Lens transmission is defined as the light intensity fraction of a narrow beam that passes through the lens along its optical axis. ${ }^{18,19}$ The assessment of the light transmission of the ocular lens is derived from a principle suggested by Zeimer and Noth. ${ }^{17}$ It is based on the assumption that the peak quantum efficiency for autofluorescence is about equal in the anterior and posterior part of the lens since the lens has a symmetric structure consisting of homogeneous layers around its nucleus. ${ }^{20}$ Consequently, any difference in fluorescence intensity between both parts can be attributed to a loss of exciting as well as fluorescent light by scatter and by absorption in the lens nucleus and cortex. The lens transmission for $\lambda=450$ to $550 \mathrm{~nm}, T$, can then be approximated by the equation

$$
T=\sqrt{F_{p} / F_{a}}
$$

where $F_{a}$ and $F_{p}$ are the values of the relative peak autofluorescence in the anterior and posterior part of the lens, respectively (Figure 1). From this equation, it follows that a certain transmission value can occur with a low as well as with a high average lens autofluorescence value. The validity of the assumption concerning an equal fluorescence quantum efficiency in the anterior and posterior part of the lens was verified by in vitro measurements of peak fluorescence efficiency in both parts of donor lenses $(N$ $=7$, age 24 to 85 years). ${ }^{18}$ Note that the lens transmission can be measured in vivo in a few seconds with no burden to the patient.

\section{StATISTICAL EVAlUATION}

\subsection{EVALUATION OF FLUOROPHOTOMETRIC RESULTS}

Several statistical procedures were used to evaluate fluorophotometric results. The lens autofluorescence values of healthy volunteers as a function of age could be approximated by a linear function with the use of a weighted linear regression procedure using squared inverse age as a weighting function. ${ }^{3}$ The values of lens transmission in healthy volunteers as a function of age could be approximated by a single exponential decay ${ }^{18}$ with the use of a least-squares approximation procedure based on the gradient expansion algorithm of Marquardt. ${ }^{21}$

\subsection{NORMALIZATION FOR NORMAL AGE DEPENDENCY}

To make a comparison of lens autofluorescence or transmission values among various (patient) groups possible, each value was normalized for normal age dependency. The normalization was done by taking the ratio between the value measured and the value expected for a healthy control of the same age. This expected value was calculated 


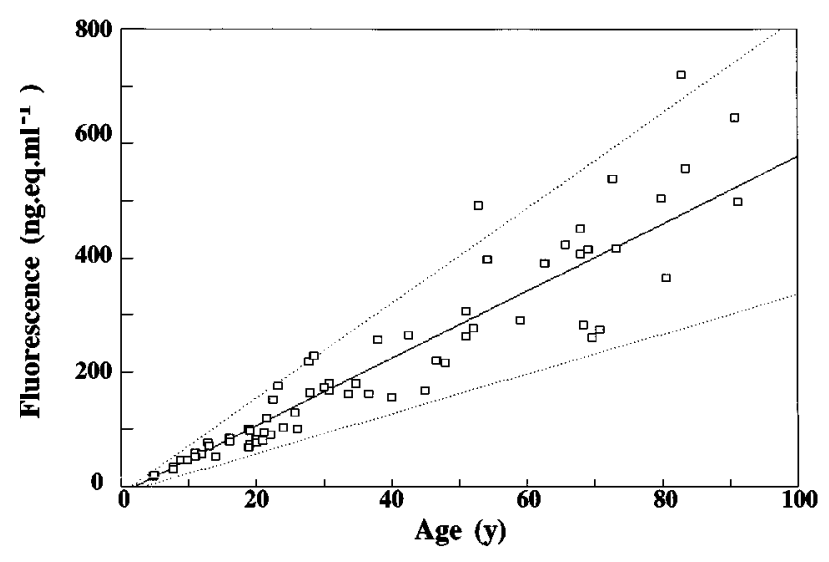

Fig. 2 Autofluorescence of the lens of healthy volunteers as a function of age. Each square represents the average value of both eyes. The solid and dotted lines represent the mean value and the 95\% probability limits and were obtained by a weighted linear regression procedure to the data points. The data points were obtained from Bleeker et al., Invest. Ophthalmol. Vis. Sci. 27, 791-794 (1986) and van Wirdum et al., Graefe's Arch. Clin. Exp. Ophthalmol. 227, 26-29 (1989).

using an approximating function describing the values for healthy controls as a function of age. A comparison between patients and age-matched healthy controls could not be used because of the large interindividual spread in the values of individuals of the same age and category.

\subsection{COMPARISON AMONG GROUPS}

The Student two-tailed $t$ test was used to evaluate the significance level between two groups if the age-normalized values were normally distributed; otherwise the nonparametric Mann-Whitney test with corrections for tied ranks was used. ${ }^{22}$ Normal distribution was assessed with the d'Agostino test for departure of normality. ${ }^{22}$

\section{RESULTS}

\subsection{LENS AUTOFLUORESCENCE OF HEALTHY INDIVIDUALS}

The autofluorescence of the anterior part of the lens as a function of age is presented for healthy volunteers in Figure 2.,23 These volunteers were recruited from co-workers and their families in Leiden, The Netherlands; had no general diseases; and showed no ocular diseases on slit lamp examination. The solid line in the figure corresponds with the course of the average lens autofluorescence as a function of age. The spread in the values was found to increase linearly with age and was accounted for in the statistical evaluation. Each point in the figure represents the mean value of both eyes since the values of the left and right eyes were found to be strongly correlated. ${ }^{3}$ The regression procedure revealed that the expected value of lens fluorescence,

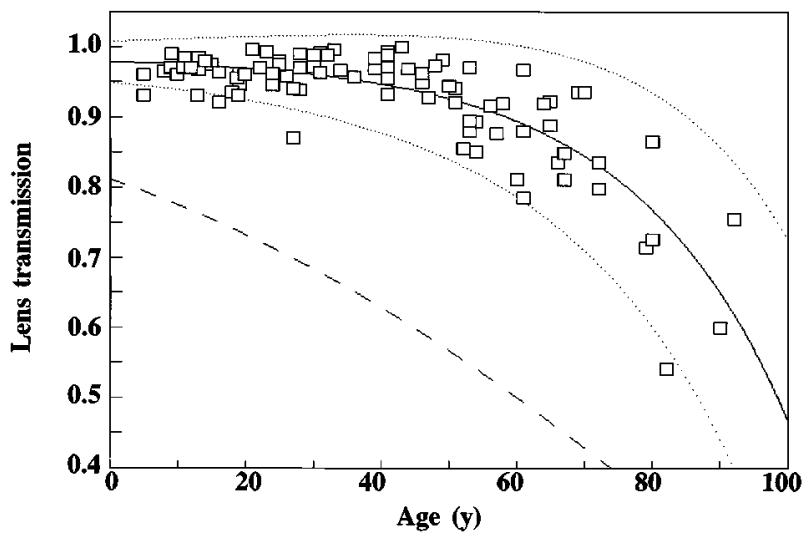

Fig. 3 Lens transmission $(\lambda=450$ to $550 \mathrm{~nm}$ ) in healthy volunteers as a function of age. Each square represents the average value of both eyes. The solid and dotted lines represent the mean value and $90 \%$ probability limits and were obtained by a least-squares approximation procedure to a single exponential decay. The broken line corresponds to the mean transmission value of donor lenses as calculated from the data of Weale [R. A. Weale, J. Physiol. (Lond.) 395, 577-587 (1988)]. The data points were obtained from van Best et al., Invest. Ophthalmol. Vis. Sci. 26, 532-536 (1985) and van Wirdum et al., Graefe's Arch. Clin. Exp. Ophthalmol. 227, 26-29 (1989).

$F_{a}$ (in ng.eq.fluorescein. $\mathrm{ml}^{-1}$ ), of a healthy control can be related to age $A$ (in years) by the equation:

$$
F_{a}=F_{0}+F_{s} \times A
$$

$F_{0} \quad$ was $\quad-12.1$ ng.eq. $\mathrm{ml}^{-1}$ and $F_{s}$ was 5.9 ng.eq. $\mathrm{ml}^{-1} \cdot \mathrm{y}^{-1}$. The correlation coefficient was 0.96 and the slope of the line differed significantly from zero $(P<0.0001)$. The value of $F_{0}$ differed significantly from zero as well $(p=0.002)$ and corresponded to an increase in lens autofluorescence starting at the age of 2 .

Similar measurements using an identical fluorophotometer were also performed in Coimbra, Portugal. ${ }^{12}$ The sensitivities of the instruments in Leiden and in Coimbra were checked by measuring the autofluorescence values of the lenses of the same volunteer. In Coimbra, $F_{0}$ was -62.2 ng.eq. $\mathrm{ml}^{-1}$ and $F_{s}$ was 9.14 ng.eq. $\mathrm{ml}^{-1} \cdot \mathrm{y}^{-1}$. The correlation coefficient was 0.86 . The regression line of the Portuguese controls was significantly higher $(p<0.001)$ but just within 2 SD of that of the Dutch controls. A similar relationship has been found for a third group in California: ${ }^{24} \quad F_{0}$ was -63.5 ng.eq. $\mathrm{ml}^{-1}$ and $F_{s}$ was 8.0 ng.eq. $\mathrm{ml}^{-1} \cdot \mathrm{y}^{-1}$.

\subsection{LENS TRANSMISSION IN HEALTHY INDIVIDUALS}

The lens transmission of 93 healthy volunteers without noticeable cataract is presented as a function of age in Figure 3. ${ }^{18,23}$ Each data point represents the average value of left and right eyes since the values of both eyes were strongly correlated (correlation coefficient $0.94, p<0.0001$ ). It can be concluded that the lens transmission of healthy in- 
dividuals decreases slowly with age up to about 50 years. After this, it decreases rapidly. The transmission values could be approximated by a single exponential function:

$$
T(A)=T_{0}\left[1-\exp \left(\left(A-t_{0}\right) / t_{m}\right)\right]
$$

where $T(A)$ is the lens transmission, $A$ is age in years, and $T_{0}, t_{0}$, and $t_{m}$ are constants with values of $0.985,115$, and 23.0, respectively. These values were obtained by a least-squares, curve-fitting procedure. ${ }^{21}$ The coefficient of relative deviation was $6.1 \%$. The transmission curve is comparable to that calculated from the absorbance curve of human donor lenses obtained by R. A. Weale, ${ }^{25}$ (Figure 2, $\lambda=500 \mathrm{~nm}$ in Ref. 25; broken line in Figure 3):

$$
\begin{gathered}
T(A)=\exp (-D(A) \cdot \ln 10) \\
D(A)=D(0) \times \exp (\beta A)
\end{gathered}
$$

where $D(A)=$ absorbance of the lens, $A=$ age (years), $D(0)=0.0907$, and $\beta=0.02$. Note that Eq. (3) represents an approximating function corresponding reasonably with the values measured up to the age of 95 and that the number of healthy noncataractous lenses for individuals over 75 is low and may have a considerable impact on the curve fitting.

\subsection{CHECK OF THE EFFECT OF UV-A ON LENS AUTOFLUORESCENCE}

A special study on the effect of UV-A irradiation on autofluorescence of the ocular lens was performed in an animal model. ${ }^{26}$ In this study, rabbit lenses were irradiated and the lens fluorescence was determined in vivo by fluorophotometry. The light source was a slide projector containing a standard tungsten-halogen bulb $(24 \mathrm{~V}, 250 \mathrm{~W})$ and three IRblocking filters. The irradiated central lens area was about $4 \mathrm{~mm}^{2}$. The maximum power of the white light, including UV-A, (Figure 4) on the lens was 1.2 $\mathrm{W} \mathrm{cm}{ }^{-2}$. By means of a UV bandpass filter, a maximum UV-A power of $0.015 \mathrm{~W} \mathrm{~cm}^{-2}$ could be delivered on the lens. The eyes were exposed for $15 \mathrm{~min}$ up to $180 \mathrm{~min}$.

The lens autofluorescence was found to increase linearly with the UV-A energy (Figure 5; correlation coefficient $0.86 ; p<0.0001$ ). The increase amounted to 0.041 ng.eq. $\mathrm{ml}^{-1}$ per J.cm ${ }^{-2}$. A significant increase in autofluorescence was observed only if UV-A was present $(p<0.005)$. The simultaneous exposure to high-intensity white light $\left(1.2 \mathrm{~W} / \mathrm{cm}^{-2}\right.$ of white light to about $0.007 \mathrm{~W} / \mathrm{cm}^{-2}$ of UV-A) did not alter the effect of the UV-A light (Figure 5). The increased autofluorescence values were found to return to normal within 4 days. Note that the intercept of the regression line in the figure did not differ significantly from the autofluorescence of the nonexposed rabbit lens, indicating the absence of a threshold energy for lens alterations by UV-A.

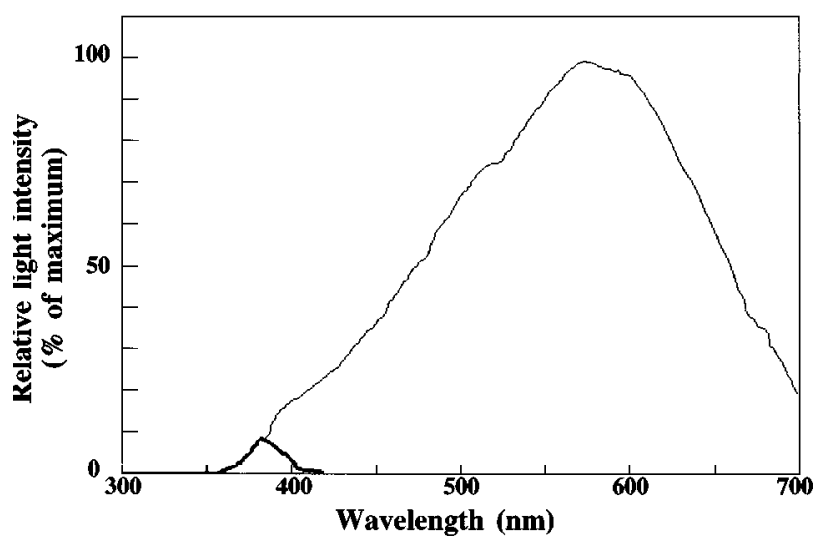

Fig. 4 Relative light intensity as a function of the wavelength of the tungsten halogen lamp used to irradiate rabbit lenses. The thin line represents the total spectrum (excluding IR) and the thick line the UV-A spectrum as obtained with a bandpass filter. The data were obtained from van Vreeswijk et al., Exp. Eye Res. 56, 349-354 (1993).

These results clearly demonstrated that UV-A light significantly increases lens autofluorescence in the rabbit.

\subsection{LENS AUTOFLUORESCENCE OF DIABETES PATIENTS}

The lens autofluorescence values of insulindependent diabetes patients is presented as a function of age in Figure $6 .^{3,23}$ These patients were recruited from the outpatient department of the clinic, had no disease except diabetes, and no signs of cataract on slit lamp examination. The relationship of the anterior lens autofluorescence $\left(F_{a}\right.$ in nanogram equivalents of fluorescein $\mathrm{ml}^{-1}$ ) with age

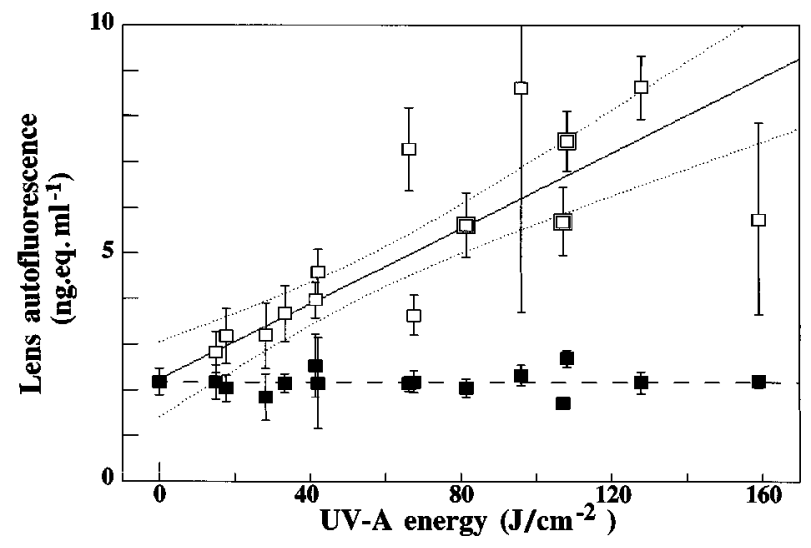

Fig. 5 Autofluorescence of rabbit lenses as a function of the UV-A energy delivered. Open and solid squares: irradiated eyes and nonirradiated contralateral eyes, respectively. Solid and dotted lines: mean value and $95 \%$ probability limits for the line obtained with a linear regression procedure to the data points representing irradiated eyes. Broken line: mean value of the nonirradiated eyes. Bars: standard deviation of the measurements. Double squares: eyes that were irradiated concomitantly with white light $113 \mathrm{~kJ}$ $\mathrm{cm}^{-2}$ ). The data were obtained from van Vreeswijk et al., Exp. Eye Res. 56, 349-354 (1993). 


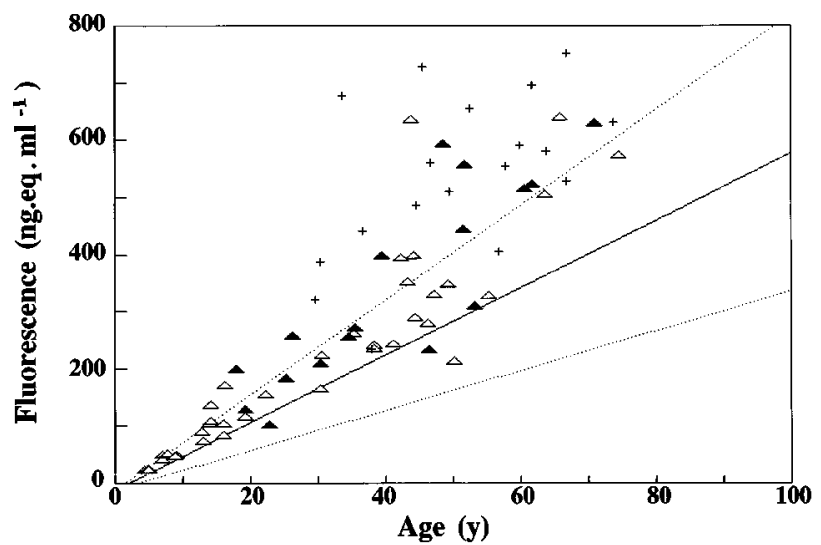

Fig. 6 Autofluorescence of the lens as a function of age in diabetic patients. A subdivision was made to the duration of diabetes: open triangles, <6 years; solid triangles, 6 to 15 years; crosses, $>15$ years. Solid and dotted lines: mean value and $95 \%$ probability limits for healthy controls. The data points were obtained from Bleeker et al., Invest. Ophthalmol. Vis. Sci. 27, 791-794 (1986) and van Wirdum et al., Graefe's Arch. Clin. Exp. Ophthalmol. 227, 26-29 (1989).

( $A$ in years) and diabetes duration ( $D$ in years) was investigated by multiple regression analysis with the same weighting system used for the healthy controls. The analysis revealed the following equation:

$$
F_{a}=-69.3+7.98 \times A+8.78 \times D .
$$

The coefficient for age and that for duration of diabetes differed from zero $(7.98, p<0.001$ and 8.78 , $p<0.001$, respectively). This is illustrated in Figure 6 by using different symbols for three groups according to duration of diabetes. Roughly speaking, one can say that each year of diabetes corresponds with one extra year of age.

The value of lens autofluorescence for each individual was normalized for normal age dependency by calculating the ratio between the value measured and the value calculated using Eq. (2) (Table 1). The ratios of the three diabetes groups differed significantly from the ratios of the healthy controls (Mann-Whitney test, $p \leqslant 0.001$ ). The differences among the diabetes groups were also significant $(p<0.006)$ except between patients with a diabetes duration of less than 6 years and those between 6 and 15 years, respectively $(p>0.2)$.

\subsection{LENS TRANSMISSION OF DIABETIC PATIENTS}

The lens transmission values of diabetic patients are presented as a function of age in Figure 7. ${ }^{9,23}$ It can be seen that the deviation from the values for healthy controls (solid line in the figure) is larger for the group who had diabetes in excess of 10 years (squares) than for the group with a lower duration (triangles). One can roughly estimate that the sharp decrease in transmission value in patients
Table 1 Lens autofluorescence of diabetic patients.

\begin{tabular}{|c|c|c|c|}
\hline \multirow[b]{2}{*}{ Group } & \multicolumn{3}{|c|}{ Age-normalized ${ }^{a}$ lens autofluorescence } \\
\hline & Number & Mean \pm SD & $P$ value ${ }^{b}$ \\
\hline $\begin{array}{l}\text { Diabetics }^{\mathrm{C}} \\
\quad \text { (duration }<6 \text { years) }\end{array}$ & 38 & $1.40 \pm 0.34$ & $<0.0001$ \\
\hline $\begin{array}{l}\text { Diabetics }^{c} \\
\quad \text { (duration 6-15 years) }\end{array}$ & 17 & $1.48 \pm 0.39$ & 0.0001 \\
\hline $\begin{array}{l}\text { Diabetics }^{c} \\
\quad \text { (duration > } 15 \text { years) }\end{array}$ & 19 & $1.95 \pm 0.57$ & $<0.0001$ \\
\hline Healthy controls & 66 & $1.00 \pm 0.22$ & - \\
\hline
\end{tabular}

a Value measured divided by value calculated for a healthy control of the same age.

b Two-sided Mann-Whitney test between patient group and healthy controls.

${ }^{c}$ Insulin-dependent diabetes mellitus patients.

with diabetes in excess of 10 years occurs about 15 years earlier than in healthy controls. ${ }^{19}$ The lens transmission values normalized for normal age dependency with the use of Eq. (3) are presented in Table 2. The values of the patient groups differed from those of healthy controls (Mann-Whitney test, $P<0.02)$. The difference between both patient groups was also significant $(p<0.001)$.

\subsection{LENS AUTOFLUORESCENCE AND TRANSMISSION IN GLAUCOMA OR OCULAR HYPERTENSION PATIENTS}

The lens autofluorescence and transmission values of patients with newly detected, untreated, primary open-angle glaucoma (POAG; patients with optic nerve damage, visual field defects and an ocular pressure above $21 \mathrm{~mm} \mathrm{Hg}$ ) or ocular hypertension

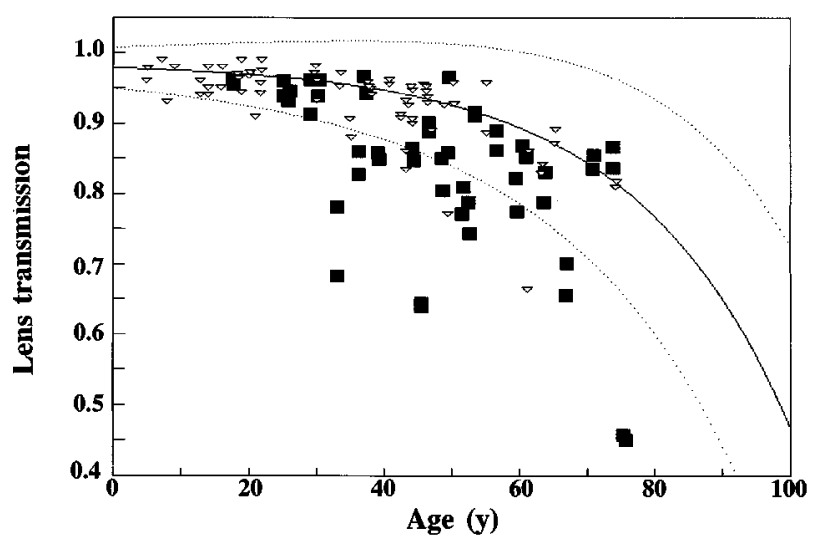

Fig. 7 Lens transmission for blue-green light as a function of age in diabetic patients. A subdivision was made to the duration of diabetes: triangles, $\leqslant 10$ years; squares, $>10$ years. Solid and dotted lines: mean value and $95 \%$ probability limits for healthy controls. The data points were obtained from van Best et al., Invest. Ophthalmol. Vis. Sci. 26, 532-536 (1985) and van Wirdum et al., Graefe's Arch. Clin. Exp. Ophthalmol. 227, 26-29 (1989). 
Table 2 Lens transmission of diabetic patients.

\begin{tabular}{lccc}
\hline & \multicolumn{2}{c}{ Age-normalized ${ }^{a}$ lens transmission } \\
\cline { 2 - 5 } & Number & Mean \pm SD & $P$ value $^{b}$ \\
Group & 68 & $0.98 \pm 0.045$ & 0.024 \\
$\begin{array}{l}\text { Diabetics } \\
\text { (duration } \leqslant 10 \text { years) }\end{array}$ & 50 & $0.91 \pm 0.113$ & $<0.0001$ \\
$\begin{array}{l}\text { Diabetics } \\
\text { (duration }>10 \text { years) }\end{array}$ & 93 & $1.00 \pm 0.056$ & - \\
\hline \begin{tabular}{l} 
Healthy controls \\
\hline
\end{tabular}
\end{tabular}

a Value measured divided by value calculated for a healthy control of the same age.

b Two-sided Mann-Whitney test between patient group and healthy controls.

c Insulin-dependent diabetes mellitus patients.

(OHT; patients with intraocular pressure above 21 $\mathrm{mm} \mathrm{Hg}$ ) were compared with the values for healthy controls. ${ }^{27}$ All fluorescence and transmission values were normalized for age dependency by taking the ratios between the measured values for each participant and the calculated values for a healthy control of the same age [Eqs. (2) and (3)]. The results are presented in Tables 3 and 4 . The mean agenormalized autofluorescence and transmission values for the POAG and the OHT patients did not differ significantly from the values for healthy controls (Student two-tailed $t$ test; $p \geqslant 0.2$ ).

\section{DISCUSSION}

\subsection{HEALTHY CONTROLS}

The increase in lens autofluorescence $(\lambda=530 \mathrm{~nm})$ in healthy controls starting from early childhood and continuing during life supports theories dealing with UV light-induced photochemical generation of fluorescent proteins, a process likely to start directly after birth. The $55 \%$ higher increase in lens

Table 3 Lens autofluorescence of glaucoma and ocular hypertension patients.

\begin{tabular}{lccc} 
& \multicolumn{3}{c}{ Age-normalized ${ }^{\text {a }}$ lens autofluorescence } \\
\cline { 2 - 4 } Group & Number & Mean \pm SD & $P$ value $^{b}$ \\
\hline $\begin{array}{l}\text { Glaucoma patients } \\
\text { c }\end{array}$ & 16 & $1.06 \pm 0.27$ & 0.6 \\
$\begin{array}{l}\text { Ocular hypertension } \\
\text { patients }\end{array}$ & 22 & $1.04 \pm 0.25$ & 0.7 \\
Healthy controls & 24 & $1.01 \pm 0.25$ & - \\
\hline $\begin{array}{l}\text { a Value measured divided by value calculated for a healthy control of the } \\
\text { same age. }\end{array}$ & & \\
b Two-sided Student $t$ test between patients and healthy control groups. \\
c Untreated open-angle glaucoma patients. \\
d Untreated ocular hypertension patients.
\end{tabular}

Table 4 Lens transmission of glaucoma and ocular hypertension patients.

\begin{tabular}{|c|c|c|c|}
\hline \multirow[b]{2}{*}{ Group } & \multicolumn{3}{|c|}{ Age-normalized ${ }^{a}$ lens transmission } \\
\hline & Number & Mean \pm SD & $P$ value ${ }^{b}$ \\
\hline Glaucoma patients $^{c}$ & 16 & $0.96 \pm 0.08$ & 0.3 \\
\hline Ocular hypertension patients ${ }^{d}$ & 22 & $0.96 \pm 0.09$ & 0.2 \\
\hline Healthy controls & 24 & $1.00 \pm 0.14$ & - \\
\hline \multicolumn{4}{|c|}{$\begin{array}{l}\text { a Value measured divided by value calculated for a healthy control of the } \\
\text { same age. }\end{array}$} \\
\hline \multicolumn{4}{|c|}{ b Two-sided Student $t$ test between patients and healthy control groups. } \\
\hline \multicolumn{4}{|c|}{ c Untreated open-angle glaucoma patients. } \\
\hline d Untreated ocular hypertension $\mathrm{pc}$ & tients. & & \\
\hline
\end{tabular}

autofluorescence as a function of age in the Portuguese group in comparison with the Dutch group may be attributed to an increased exposure to UV radiation in the Portuguese population since Coimbra is situated at a lower latitude than Leiden ( 40 deg and 52 deg northern latitude, respectively). The susceptibility to UVA found in vivo in rabbit lenses tends to confirm this assumption and provides further evidence that low-intensity UV-A exposure to the human lens should be avoided, especially when taking into account the probability that the primate lens is more susceptible to UV-A than the rabbit lens.

The lens transmission was found to remain constant up to the age of 50 and started to decrease hereafter. The lower transmission values in donor lenses found by Weale may be attributed to the enucleation process and the postmortem period.

\subsection{PATIENTS}

The increase of lens autofluorescence value as a function of age was significantly higher in diabetes patients than in healthy controls. The extra increase in fluorescence as a result of 1 year of diabetes was about as large as 1 year of aging in healthy controls. The decrease in lens transmission values in diabetes patients occurred about 15 years earlier than in healthy controls. This study supports the assumption that diabetes is an important risk factor for the development of cataract and that this risk factor becomes more important the longer one has diabetes.

The mean age-normalized lens autofluorescence and transmission values of the untreated POAG and untreated OHT patients differed less than 5\% from those of healthy controls $(P \geqslant 0.2)$. This implies that neither untreated glaucoma nor ocular hypertension are associated with an increased risk of developing cataract in the axial region of the lens. Changes outside this region may not be evident since fluorophotometry scans only a small region along the optical axis of the eye. The results are supported by those of a previous study ${ }^{28}$ in which 
the incidence of cataract in a healthy and a glaucoma population treated with pilocarpine or carbacholine only did not differ significantly.

The results presented here contrast with those of another study in which glaucoma was identified as a powerful and independent risk factor for cataract and was held responsible for $5 \%$ of all cataracts reported in England. ${ }^{29}$ However, this study concerned medically and/or surgically treated glaucoma patients, which may have biased the study outcome. Some topical antiglaucomatous medicaments such as strong miotics were held responsible for inducing cataract. ${ }^{30,31}$ Surgical drainage procedures also have been recognized as a factor contributing to the development of cataract ${ }^{28,31-35}$ or as a cataract accelerating factor. $^{36}$

\section{CONCLUSIONS}

The studies presented here demonstrate the clinical value of fluorophotometry of the ocular lens. Fluorophotometry is a noninvasive measurement performed by nonspecialized personnel in a few seconds. The measurement is painless, easy to undergo, does not require the use of drugs or contact lenses, and does not tire or alarm the patient. It provides a quantitative evaluation of the probability for developing cataract. The results show that age, exposure to UV-A light, and diabetes are risk factors. On the other hand, the results demonstrate that having untreated OHT or POAG may not be a risk factor for developing cataract, while the treatment of the disease probably is.

\section{REFERENCES}

1. A. C. Sen, N. Ueno, and B. Chakrabarti, "Studies on human lens: I. Origin and development of fluorescent pigments," Photochem. Photobiol. 55(5), 753-764 (1992).

2. C. F. Wannemacher and A. Spector, "Protein synthesis in the core of calf lens," Exp. Eye Res. 7, 623-625 (1968).

3. J. C. Bleeker, J. A. van Best, L. Vrij, E. A. van der Velde, and J. A. Oosterhuis, "Autofluorescence of the lens in diabetic and healthy subjects by fluorophotometry," Invest. Ophthalmol. Vis. Sci. 27, 791-794 (1986).

4. M. Bando, A. Nakajima, and K. Satoh, "Coloration of human lens protein," Exp. Eye. Res. 20, 489-492 (1975).

5. J. D. Goosey, J. S. Zigler, and J. H. Kinoshita, "Crosslinking of lens crystallins in a photodynamic system: a process mediated by singlet oxygen," Science 208, 1278-1280 (1980).

6. U. P. Andley, P. Sutherland, J. N. Liang, and B. Chakrabarti, "Changes in tertiary structure of calf-lens $\alpha$-crystallin by near-UV irradiation: role of hydrogen peroxide," Photochem. Photobiol. 40, 343-349 (1984).

7. M. Kono, K. Mandal, and B. Chakrabarti, "Structure and stability of $\gamma$-crystallins-V. Covalent and non-covalent protein-protein interactions in photosensitized reactions," Photochem. Photobiol. 47, 593-597 (1988).

8. S. Lerman, J. F. Kuck, Jr., R. F. Borkman, and E. Sakar, "Induction, acceleration and prevention (in vitro) of an aging parameter in the ocular lens," Ophthalmic Res. 8, 213-226 (1976).

9. S. Lerman, B. S. Yamanishi, R. A. Palmer, J. C. Roark, and R. Borkman, "Photoacoustic, fluorescence and light transmission spectra of normal, aging and cataractous lenses," Ophthalmic Res. 10, 168-176 (1978).

10. P. M. Haard, M. Van, H. J. Hoenders, and H. C. J. Ketelaars, "Human lens nuclear cataract: hydrogen peroxide action and anthranilic acid association with lens proteins," Ophthalmic Res. 12, 252-255 (1980).

11. M. A. Mosier, J. R. Occhipinti, and N. L. Burstein, "Autofluorescence of the crystalline lens in diabetes," Arch Ophthalmol. 104, 1340-1343 (1986).

12. E. van Wirdum, M. C. Mota, J. A. van Best, E. Leite, J. P. Kappelhof, J. R. Faria de Abreu, L. C. Paul, J. Freitas Martins, J. A. Oosterhuis, and A. Marques, "Lens transmission and autofluorescence in renal disease," Ophthalmic Res. 20, 317-326 (1988).

13. M. C. Yappert, D. Borchman, and W. C. Byrdwell, "Comparison of specific blue and green fluorescence in cataractous versus normal human lens fractions," Invest. Ophthalmol. Vis. Sci. 34, 630-636 (1993).

14. G. Klang, "Measurements and studies of the fluorescence of the human lens in vivo," Acta Ophthalmol. Suppl. 31, 1-157 (1948).

15. S. Lerman, O. Hockwin, and V. Dragomirescu, "In vivo lens fluorescence photography," Ophthalmic Res. 13, 224-228 (1981).

16. S. Lerman and O. Hockwin, "Ultra-violet visible slit lamp densitography of the human eye," Exp. Eye Res. 33, 587-596 (1981).

17. R. C. Zeimer and J. M. Noth, "A new method of measuring in vivo lens transmission, and study of lens scatter, fluorescence and transmittance," Ophthalmic Res. 16, 246-255 (1984).

18. J. A. van Best, E. W. S. J. Tjin, A. Tsoi, J. P. Boot, and J. A. Oosterhuis, "In vivo assessment of lens transmission for blue-green light to autofluorescence measurements," Ophthalmic Res. 17, 90-95 (1985).

19. J. A. van Best, L. Vrij, and J. A. Oosterhuis, "Lens transmission of the blue-green light in diabetes measured by autofluorophotometry," Invest. Ophthalmol. Vis. Sci. 26, 532-536 (1985).

20. D. D. Donaldson, The Crystalline Lens, Vol. 5, Atlas of Diseases of the Anterior Segment of the Eye, pp. 1-12, Mosby, St. Louis (1976).

21. P. B. Bevington, Data Reduction and Error Analysis for the Physical Sciences, Chapter 11, pp. 204-246, McGraw-Hill, New York (1969).

22. J. H. Zar, "The normal distribution," and "Two-sample hypotheses," pp. 79-96 and 122-149 in Biostatistical Analysis, B. Kurtz, Ed., Prentice-Hall, Englewood Cliffs, NJ (1984).

23. E. van Wirdum, J. A. van Best, G. J. Bruining, C. de Beaufort, and J. A. Oosterhuis, "Blood-retinal and blood-aqueous barrier permeability, lens autofluorescence and transmission in insulin-dependent diabetic youngsters," Graefe's Arch. Clin. Exp. Ophthalmol. 227, 26-29 (1989).

24. J. R. Occhipinti, M. A. Mosier, and N. Burstein, "Autofluorescence and light transmission in the aging crystalline lens," Ophthalmologica 192, 203-209 (1986).

25. R. A. Weale, "Age and the transmittance of the human crystalline lens," J Physiol. (Lond.) 395, 577-587 (1988).

26. H. van Vreeswijk, E. P. M. Boets, and J. A. van Best, "The effect of white light and UV-A on the green autofluorescence of the rabbit lens in vivo," Exp. Eye Res. 56, 349-354 (1993).

27. E. V. M. J. Kuppens, J. A. van Best, and C. C. Sterk, "Is glaucoma associated with an increased risk of cataract?," Br. J. Ophthalmol. 79, 649-652 (1995).

28. R. N. Shaffer and G. Rosenthal, "Comparison of cataract incidence in normal and glaucomatous population," Am. J. Ophthalmol. 69, 740-746 (1970).

29. J. J. Harding, M. Egerton, R. van Heyningen, and R. S. Harding, "Diabetes, glaucoma, sex, and cataract: analysis of combined data from two case control studies," Br. J. Ophthalmol. 77, 2-6 (1993).

30. U. Axelsson and A. Holmberg, "The frequency of cataract after miotic therapy," Acta Ophthalmol. 69, 740-746 (1966).

31. W. E. Layden, "Cataracts and glaucoma," in Duane's Clinical Ophthalmology, pp. 1-22, W. Tasman and E. A. Jaeger, Eds., Rev. ed., Lippincott-Raven, Philadelphia-New York (1979).

32. H. S. Sugar, "Postoperative cataract in successfully filtering glaucomatous eyes," Am. J. Ophthalmol. 69, 740-746 (1970).

33. E. L. Greve, C. L. Dake, J. H. J. Klaver, and E. M. G. Mut- 
saerts, "Ten year prospective follow-up of a glaucoma operation," Int. Ophthalmol. 8, 139-146 (1985).

34. J. J. Harding, R. S. Harding, and M. Egerton, "Risk factors for cataract in Oxfordshire: diabetes, peripheral neuropathy, myopia, glaucoma, and diarrhoea," Acta Ophthalmol. 67, 510-517 (1989).
35. L. Laatikainen, "Late results of surgery on eyes with primary glaucoma and cataract," Acta Ophthalmol. 49, 281-292 (1971).

36. P. G. Watson, C. Jakeman, M. Ozturk, M. F. Barnett, F. Barnett, and K. T. Khaw, "The complications of trabeculectomy (a 20-year follow-up)," Eye 4, 425-438 (1990).

Acknowledgment: The Publishers wish to acknowledge permission received from publishers and authors to reproduce figures used in this paper.

250 Journal of Biomedical Optics • July 1996 • Vol. 1 No. 3 